\title{
Longitudinal rates of smoking in a schizophrenia sample
}

\author{
J 0 Goldberg, J Van Exan
}

Department of Psychology, York University, North York, Ontario, Canada

Correspondence to:

$\mathrm{J} 0$ Goldberg, Department of Psychology, York University, 4700 Keele St., North York, Ontario, Canada M3J 1P3; jgoldber@yorku.ca

Received 17 January 2008 Accepted 17 May 2008 Published Online First 3 June 2008

\section{ABSTRACT}

Objectives: Despite the well documented link between high rates of smoking and schizophrenia, there have been no longitudinal studies that have looked at rates of smoking and associated factors over time. This prospective study examined the longitudinal rates of smoking in a schizophrenia clinic sample over a decade.

Methods: Longitudinal survey research was conducted in a well established community-based psychiatric rehabilitation program in Hamilton, Ontario, Canada, providing long-term intensive case management and rehabilitation skills training. Stable community outpatients diagnosed with schizophrenia were surveyed initially in 1995 $(n=102)$ and then resurveyed 11 years later in 2006 $(n=76)$. The main outcome measure was self-report of smoking status.

Results: Smoking rates dropped significantly over time, with evidence that the number of "quitters" tripled over the past decade and the number of "everyday" smokers decreased by almost a third from $63.2 \%$ down to $43.3 \%$ $(p<0.001)$.

Conclusions: The findings from the present study suggest that it is possible to obtain reduced smoking prevalence over time in a selected schizophrenia outpatient sample, though further research is required to better understand the factors related to quitting smoking in individuals with schizophrenia.

The rates of smoking over the past decade have declined in national population-based samples in Western countries, but the trends in rates of smoking over time among severe mental illness populations have not been studied to date, even though people with psychiatric disorders such as schizophrenia have been considered especially vulnerable. ${ }^{1}$ For example, in the Canadian province of Ontario, where the current research was conducted, the prevalence of smoking has decreased in the general population from $28.2 \%$ in 1995 to $20.7 \%$ in 2005. ${ }^{2}$ By contrast, the prevalence of smoking in individuals with persistent mental illness is significantly higher than the general population when examined using crosssectional data, ${ }^{145}$ but the rates over time are unknown. Individuals diagnosed with schizophrenia, ${ }^{67}$ schizoaffective disorder, ${ }^{6}$ bipolar disorder, ${ }^{8}$ panic disorder ${ }^{9}$ and depression ${ }^{6}$ have all been found to be generally more susceptible to greater tobacco use than the general population. Of particular relevance is a cross-sectional survey examining the prevalence of smoking in patients with schizophrenia, which found that $61 \%$ of people with schizophrenia reported being current tobacco users and this was found to be approximately 2.5 times the rate found in other diagnostic categories, ${ }^{6}$ though prevalence rates do vary worldwide. ${ }^{7}$ People with severe mental illness have also been found to be approximately one-fifth as likely as the general population to stop smoking. ${ }^{10}$ Additionally, the high rates of smoking in individuals with schizophrenia places them at increased risk of mortality ${ }^{112}$ and as vulnerable "targets" for the tobacco industry. ${ }^{13}$ What is still unclear from the literature, since there have been no longitudinal studies to date, is whether the rates of smoking change in patients with schizophrenia over time and this is a primary focus of the research discussed below.

The current literature on the neurobiology of schizophrenia and nicotine addiction has advanced greatly over the past decade and has proposed mainly biological models to explain the high prevalence of tobacco use in people with schizophrenia. ${ }^{14}$ A prominent explanation for the association between smoking and schizophrenia has been the self-medication hypothesis that suggests individuals with schizophrenia use tobacco to compensate for cognitive deficits and/or negative symptoms attributed to schizophrenia. ${ }^{1-16}$ In recent years, specific nicotinic receptors in the brain have been identified and people with schizophrenia have been found to have a decreased expression of low and high affinity nicotinic receptors, suggesting that people with schizophrenia smoke at higher rates due to deficits in nicotinic receptor systems that are important for cognitive functioning. ${ }^{17}$ The first generation drug treatments for schizophrenia were generally found to be effective in reducing positive symptoms, but less effective in reducing negative symptoms associated with schizophrenia and, thus, researchers hypothesised that individuals with schizophrenia were smoking to reduce the side-effects of these drugs. ${ }^{17}$ With the advent of the second generation antipsychotic treatments available to manage schizophrenia symptoms including negative symptoms, prescription patterns have changed dramatically over the past decade. The advantages of second generation antipsychotics, such as clozapine, risperidone and olanzapine, in reducing negative symptoms associated with schizophrenia was also thought to subsequently help reduce the high smoking rates in people with schizophrenia. ${ }^{18}$ Reduced rates of smoking in schizophrenia have been identified when patients are switched from first generation antipsychotics to clozapine, but more research is needed to clearly delineate the underlying mechanisms related to the association between smoking and schizophrenia. ${ }^{19} 20$

There has been a proliferation of research on the determinants of smoking cessation and the efficacy 
of smoking cessation programs that target biological and psychological aspects of smoking behaviour using general population samples. ${ }^{21}$ Research that has examined the efficacy of these programs in populations with schizophrenia has generally found that the "quit" rates are comparably low across psychiatric and non-psychiatric populations. ${ }^{22-24}$ It is still unclear whether specialised programs are required to address the unique needs of individuals with schizophrenia. ${ }^{24}$ The site of the present study, the Hamilton Program for Schizophrenia (HPS), has developed and incorporated the manualised "Smokebusters" curriculum ${ }^{24}$ into its rehabilitation work for more than 15 years, making the group-based behaviourallyoriented strategies available to interested outpatients; this program incorporates the stages of change model as well as information from current research on smoking and mental illness. ${ }^{25}$

This study addressed the gaps noted in the literature by conducting a survey investigation of smoking habits using naturalistic longitudinal data from a clinic sample of outpatients with persistent residual symptoms of schizophrenia. ${ }^{26}$ The main purpose was to investigate whether there is evidence of changes in long-term tobacco use outcomes for outpatients with schizophrenia. We hypothesised that there would be a decline in the rates of smoking over time (more than a decade) in a schizophrenia clinic sample, that might mirror the declining rates in the general population (coinciding with societal smoking restrictions), and sought further to examine what factors differentiated "everyday" smokers who quit and those who did not quit. Specifically, we were interested in examining whether a "switch" from first generation to second generation antipsychotic medications, as well as participation in a specialised group-based smoking management program, might be associated with reduced rates of smoking.

\section{METHODS}

\section{Participants and settings}

The sample consisted of outpatients from HPS, a communitybased psychiatric rehabilitation program. ${ }^{26}{ }^{27}$ The program follows an intensive case management model ${ }^{28}$ in which each outpatient has an assigned case manager with whom they interact on a frequent basis over many years of support and are comfortable and reliable in self-reporting information. The first wave of the survey was conducted between August 1995 and October 1995. Potential participants were told that they would be completing a self-report survey that was designed to obtain information regarding smoking habits, their stage of readiness to change their smoking behaviour, and their experience of factors that influence their smoking behaviours. Participants gave informed written consent and were assured that the information provided was confidential. The surveys were conducted through a combination of telephone and face-toface interviews. The final sample in 1995 included 102 outpatients including those who had never smoked, those who smoked in the past but quit and those who currently smoke. ${ }^{25}$

The evaluation study protocol for the second wave of the survey in 2006 was the same as the first wave and was reviewed and approved by the Research Committee of HPS project and the Community Social Vocational Rehabilitation (CSVR) Schizophrenia Board. All of the individuals who participated in the 1995 survey were contacted in April 2006 and asked to participate in a follow-up survey. Upon giving informed consent and receiving assurance about confidentiality, participants completed the second wave of the survey from May 2006 to
June 2006. In 2006, 76 (74.5\%) participants completed the survey from the initial sample of 102 participants from the 1995 sample. The following reasons were indicated for the 26 (25.5\%) participants who did not complete the 2006 survey: 14 participants were no longer in the program (13.7\%), 5 participants had died (4.9\%; three of these were never smokers, while two smoked every day), 5 participants could not be reached (4.9\%), 1 participant had moved away (1.0\%) and 1 participant was physically unable to complete the survey $(1.0 \%)$.

\section{Procedures and measures}

In order to examine long-term outcomes, the survey was administered to outpatients in 1995 (time 1) and then readministered to the same outpatients 11 years later in 2006 (time 2). The questions and the wording were identical for both surveys in order to maintain consistency. Questions on smoking habits were based on those outlined by Health Canada. ${ }^{29}$ The participant's current smoking status was based on self-report only. Specifically, participants were asked to indicate their smoking status at the "present time" (everyday, occasionally, used to smoke but quit and not at all/never have). The questionnaire asked only about smoking and not other forms of tobacco use. The 2006 survey had additional questions regarding the "Smokebusters" program that is offered at HPS (number of times attended and level of helpfulness of the program).

\section{Data analysis}

For the analyses, we used SPSS version 15.0. Data on all variables were available for all participants. Responses from 2006 were compared with responses from 1995 to check for consistency in responding. There were two participants who were excluded from the current analysis due to discrepancies found in the reported data. Specifically, these two participants reported smoking in 1995, but reported "never smoking" in the 2006 survey. A $\chi^{2}$ analysis was used to examine whether there were differences in smoking status depending on the year of participation. Specifically, this test was used to ensure that the participants who did not complete the second wave of the survey were also more likely to be current smokers. Descriptive analyses were used to examine sample characteristics in both waves of the survey including, gender, age, living situation and number of years at HPS. All further statistical analyses were conducted using only data from participants who completed both waves of the survey $(n=76)$. In order to examine whether smoking status and antipsychotic medications independently changed from 1995 to 2006, the data were analysed using the McNemar-Bowker repeated measure $\chi^{2}$ test. Analysis by $\chi^{2}$ was used to determine if a change from first generation to second generation antipsychotic medications could explain the difference between participants who were smokers in 1995 and quit in 2006 and those who were still smoking in 2006. Independent sample Student t tests were used to examine whether there was a difference in level of attendance and helpfulness of the "Smokebusters" program for participants who had quit and those who were still smoking in 2006.

\section{RESULTS}

The prevalence rates of smoking for time 1 (1995) and time 2 (2006) for participants who completed both waves of the survey are presented in table 1 . In 1995, 63.2\% of participants were current smokers, $21.1 \%$ had never smoked and $15.8 \%$ had quit smoking. In $2006,43.4 \%$ of these participants were current smokers, $19.7 \%$ had never smoked and $36.8 \%$ had quit smoking. 
Table 1 Prevalence rates grid of smoking for time 1 (1995) and time 2 (2006)

\begin{tabular}{llcccl}
\hline \multicolumn{2}{l}{ Smoking status } & & & & \\
\hline & & & & & \\
\multirow{4}{*}{1995} & Never & Quit & Everyday & Total \\
& Never & 15 & 0 & 1 & 16 \\
& Quit & 0 & 11 & 1 & 12 \\
& Everyday & 0 & 17 & 31 & 48 \\
& Total & 15 & 28 & 33 & 76 \\
\hline
\end{tabular}

The main finding was that, as predicted, smoking status (never smoked, quit smoking, everyday smoking) changed significantly over time, $\chi^{2}(2, \mathrm{n}=76)=15.22, \mathrm{p}<0.001$, with dramatic evidence that the number of "quitters" had tripled over the past decade and thus, the number of "everyday" smokers had decreased by almost a third. The descriptive characteristics for time 1 (1995) and time 2 (2006) are presented in table 2 .

The prevalence rates of type of antipsychotic medication for time 1 (1995) and time 2 (2006) for participants who completed both waves of the survey are presented in table 3 . Specifically, in 1995, 46.1\% of participants were prescribed first generation antipsychotics, while $35.5 \%$ of participants were treated with second generation antipsychotics, and $18.4 \%$ of participants were using both types of antipsychotics. In 2006 , only $9.2 \%$ of participants were being prescribed first generation antipsychotics, while $86.8 \%$ of participants were being treated with second generation antipsychotics, and $4.0 \%$ of participants were on both types of antipsychotics. In addition, a significant change from first generation to second generation antipsychotic medications was found over time, $\chi^{2}(3, \mathrm{n}=76)=38.33$, $\mathrm{p}<0.001$, demonstrating the increased psychiatric treatment using second generation antipsychotic medications in this sample in 2006.

In order to determine if a change in antipsychotic medications explained the difference between participants who were smokers in 1995 and quit in 2006 and those who were still smoking in 2006, a two-by-two $\chi^{2}$ table was calculated to see whether differences existed based on a change in the type of medication or no change in the type of psychiatric medication participants were on over time. This analysis did not include participants who were prescribed both first and second generation antipsychotic medications. There were no differ-

Table 2 Sample characteristics for time 1 (1995) and time 2 (2006)

\begin{tabular}{|c|c|c|c|c|}
\hline & & & 1995 & 2006 \\
\hline Variable & Mean (SD) & Range & n (\%) & n (\%) \\
\hline \multicolumn{5}{|l|}{ Gender: } \\
\hline Male & & & $70(68.6)$ & $52(68.4)$ \\
\hline Female & & & $32(31.4)$ & $24(31.6)$ \\
\hline Age (1995) & $35.00(6.62)$ & $20-57$ & & \\
\hline Age (2006) & $46.15(6.72)$ & $31-69$ & & \\
\hline \multicolumn{5}{|l|}{ Living situation: } \\
\hline Parental home & & & $6(5.9)$ & $4(5.3)$ \\
\hline Boarding home & & & $18(17.6)$ & $9(11.8)$ \\
\hline Supported housing & & & $16(15.7)$ & $19(25.0)$ \\
\hline Independent living & & & $59(57.8)$ & $42(55.3)$ \\
\hline Other & & & $3(2.9)$ & $2(2.6)$ \\
\hline Time at HPS (1995), years & 7.74 (4.99) & $0.03-20$ & & \\
\hline Time at HPS (2006), years & $18.66(5.44)$ & $10-33$ & & \\
\hline
\end{tabular}

HPS, Hamilton Program for Schizophrenia. ences in whether individuals changed or did not change their medication based on whether they quit smoking or were still smoking in 2006, $\chi^{2}(1)=0.615$, not significant (table 4). These findings, taken together, demonstrate that a "switch" to a different antipsychotic medication by itself does not seem to be associated with a capacity to stop smoking.

Data were also analysed to determine if differences existed in whether participants who were smokers in 1995 and who quit by 2006 were more likely to have attended the "Smokebusters" program and to have found it more helpful than those who were still smoking in 2006. The findings failed to indicate a significant association between quit rates and formal attendance in the group-based smoking management program, $(t(46)=0.97$, not significant), however, subjective ratings of high perceived helpfulness of the "Smokebusters" program were most strongly associated with successful "quitters" $(t(28)=2.06, \mathrm{p}<0.05)$. Specifically, 34 participants reported that they had attended the "Smokebusters" program. Of these respondents, $55.9 \%$ reported that it was very helpful, $41.2 \%$ reported that it was somewhat helpful and $2.9 \%$ reported that "Smokebusters" was not helpful.

Prior to analysing the results discussed above, the baseline data of the entire sample was examined to ensure that the loss of participants from the first wave of the study did not result in an attrition bias. Specifically, a $\chi^{2}$ analysis was conducted to examine whether participants who did not complete the second survey in 2006 were more likely to be smokers in the first wave of the survey in 1995 compared to participants who completed both waves of the survey. There were no significant differences in smoking status reported in 1995 (never smoked, quit smoking, everyday smoking) between participants who only completed the 1995 survey and participants who completed both surveys $\left(\chi^{2}(2, \mathrm{n}=102)=5.75\right.$, not significant $)$.

\section{DISCUSSION}

This longitudinal study found that the overall rates of smoking in a community-based psychiatric rehabilitation clinic declined significantly when examining a period of more than a decade, with evidence that the number of "quitters" has tripled and the number of "everyday" smokers decreased by almost a third. There are a few cross-sectional studies that have examined smoking cessation rates among smokers with schizophrenia and these results indicate a rather "pessimistic" view that smokers with schizophrenia are less likely to quit smoking than the general population and when compared to individuals with other severe mental illness. ${ }^{7}$ By contrast, the current findings suggests a more "optimistic" perspective that individuals with schizophrenia are in fact able to quit and indicates the necessity of examining smoking prevalence over time in schizophrenia populations in order to better understand the quit rates in this population.

A key parallel was found between the declining schizophrenia sample smoking rates over time and the declining rates of smoking in the general community. The main finding of a $31 \%$ decline in the number of daily smokers in the schizophrenia sample (from $63.2 \%$ to $43.4 \%$ ) is roughly comparable to the $27 \%$ decline witnessed in the general population (from $28.2 \%$ to $20.7 \%$ ) during the study period. Such parallel rates may imply, for schizophrenia outpatients, living in the community as they do, that the general societal tightening effects and restrictions on smoking that occurred in this past decade may have had some important impacts on them as well, including the impact of increased smoking costs and reduced visibility and availability. What is less clear is whether there are specific influences 
Table 3 Prevalence rates grid of type of antipsychotic medication for time 1 (1995) and time 2 (2006)

\begin{tabular}{|c|c|c|c|c|c|}
\hline \multicolumn{6}{|c|}{ Medication } \\
\hline & & 2006 & & & \\
\hline & & $\begin{array}{l}\text { First } \\
\text { generation }\end{array}$ & $\begin{array}{l}\text { Second } \\
\text { generation }\end{array}$ & Both & Total \\
\hline \multirow[t]{4}{*}{1995} & First generation & 6 & 29 & 0 & 35 \\
\hline & $\begin{array}{l}\text { Second } \\
\text { generation }\end{array}$ & 0 & 26 & 1 & 27 \\
\hline & Both & 1 & 11 & 2 & 14 \\
\hline & Total & 7 & 66 & 3 & 76 \\
\hline
\end{tabular}

or schizophrenia-related factors that might have contributed to the reduced rates over time.

Our study followed outpatients over a decade and found that many outpatients had been switched from first generation antipsychotic medication to predominantly second generation antipsychotic medication. Nevertheless, we failed to identify a specific association between a "switch" in medication regime by itself and change in smoking status, though the small sample size may limit the generalisability of these non-significant findings. Future longitudinal research should examine how first and second generation antipsychotic medications may differentially impact smoking behaviour in larger schizophrenia populations.

Lastly, we found that smokers who had quit by the time of the second survey reported that they perceived the "Smokebusters" program as particularly helpful compared with those who remained smokers, even though there were no significant differences regarding attendance levels of the "Smokebusters" program. This finding may be biased, since the perceived helpfulness of the "Smokebusters" program was not assessed in the first survey. Perceptions of benefit are important to highlight, however, since they demonstrate that people with schizophrenia are able to articulate and recognise the level of helpfulness of different programs. The results suggest that it is not the number of times people get help, but rather the perceived helpfulness of the programs they attend that differentiates people who quit and those who continue smoking. Further research examining what aspects of the "Smokebusters" program are most helpful is an important question for future research endeavours, particularly in light of the vulnerability of this population, ${ }^{13}$ and not to assume that individuals with severe mental illness are unable to change behaviours.

There are a number of methodological limitations in our community-based survey research. There was no control group and the sample size was small; this may have resulted in insufficient power to detect certain effects. In addition, there are limitations that exist with using survey data such as, selfreport biases and missing data though the retention rate over time $(74.5 \%)$ was good. Lastly, the sample of participants examined in our study was derived from an intensive case management clinic in which the outpatients are closely monitored (generally weekly or more) and therefore, this sample may not generalise to other settings with less frequent follow-up.

Our findings confirm that the rates of smoking in this schizophrenia population can actually decline over time. We examined whether the drop in prevalence rates could be explained by either medication regime or having access to a long-term dedicated smoking intervention, but neither factor was found to explain changes in smoking status. This suggests
Table 4 Crosstabulations of current smoking status by change in medication

\begin{tabular}{llll}
\hline Current smoking status & $\begin{array}{l}\text { No change in } \\
\text { medication }\end{array}$ & $\begin{array}{l}\text { Change in } \\
\text { medication }\end{array}$ & Total \\
\hline $\begin{array}{l}\text { Quit: } \\
\quad \text { Count }\end{array}$ & 8 & 9 & 17 \\
$\quad \begin{array}{l}\text { Expected count } \\
\text { Everyday: }\end{array}$ & 6.7 & 10.3 & \\
$\quad$ Count & & 20 & 31 \\
$\quad \begin{array}{l}\text { Expected count } \\
\text { Total: }\end{array}$ & 11 & 18.7 & \\
$\quad$ Count & 12.3 & 29 & 48 \\
\hline
\end{tabular}

that there is no single factor to explain the quit rates, but rather it is more complicated and most likely there are a number of factors involved including the effects of societal restrictions. Further research is required to better understand the factors that may influence these long-term outcomes.

\section{What this paper adds}

- Numerous cross-sectional studies have identified that individuals with schizophrenia have high rates of smoking, yet to date there have been no published longitudinal investigations of smoking rates over time.

- Over a decade ago, we surveyed a sample of stable community-based psychiatric rehabilitation clinic outpatients and replicated existing findings in the literature that smoking rates among patients with schizophrenia are more than double the rates found in the general population. The current prospective research followed-up the earlier survey more than a decade later and interviewed the same patients about their smoking status.

- Remarkably, smoking rates declined by almost a third and the number of "quitters" had tripled.

- These results challenge the rather "pessimistic" view that smokers with schizophrenia are unable to quit smoking and indicate the necessity of examining smoking prevalence over time in order to better understand the quit rates in this special needs population.

- More research is needed to help understand factors that help contribute to smoking rate decline.

Acknowledgements: The authors gratefully acknowledge the assistance provided by the Hamilton Program for Schizophrenia and Community Social and Vocational Rehabilitation (CSVR) Foundation, Anne Washington, Dr Peter E Cook, Dr Stephen List, Susan Gensey, Angela Thomas, Ashley Miles, Sandra Moll, Julie Pawlick and Bryan Miller.

Funding: Assistance was received from the CSVR Foundation, a non-profit charitable organisation that provides research funds for studies in the area of community, social and vocational rehabilitation for projects pertaining to persistent mental illness. The amount of the award was $\$ 2000$.

Competing interests: The authors declare no competing interests except as indicated elsewhere that there was assistance from the CSVR Foundation. The amount of the award was $\$ 2000$, which helped fund JVE to present portions of the paper at the 13th World Conference on Tobacco OR Health, Washington, DC, USA, 12-15 July 2006.

Ethics approval: Ethics approval was obtained for this work. Participants gave informed written consent and were assured that the information provided was confidential. 


\section{REFERENCES}

1. Prochaska JJ, Hall SM, Bero LA. Tobacco use among individuals with schizophrenia: What role has the tobacco industry played. Schizophrenia Bull 2008;34:555-67.

2. Gilmore J. Report on smoking in Canada, 1985 to 2001 (Statistics Canada Catalogue 82F0077XIE). Ottawa, Canada: Statistics Canada, 2002

3. Statistics Canada. Canadian Community Health Survey (CCHS) 3.1, 2005. Ottawa, Canada: Statistics Canada, 2005

4. Diwan A, Castine M, Pomerleau CS, et al. Differential prevalence of cigarette smoking in patients with schizophrenic vs mood disorders. Schizophrenia Res 1998:33:113-18.

5. Cuijpers $\mathbf{P}$, Smit F, ten Have $\mathbf{M}$, et al. Smoking is associated with first-ever incidence of mental disorders: a prospective population-based study. Addiction 2007:102:1303-9.

6. Morris CD, Giese AA, Turnbull JJ, et al. Predictors of tobacco use among persons with mental illnesses in a statewide population. Psych Serv 2006;57:1035-8.

7. de Leon J, Diaz FJ. A meta-analysis of worldwide studies demonstrates an association between schizophrenia and tobacco smoking behaviors. Schizophrenia Res 2005; 76:135-57.

8. Gonzalez-Pinto A, Gutierrez M, Ezcurra J, et al. Tobacco smoking and bipolar disorder. J Clin Psych 1998;59:225-8.

9. Breslau N, Klein DF. Smoking and panic attacks: an epidemiological investigation. Arch Gen Psych 1999;56:1141-7.

10. Diaz FJ, Rendon DM, Velasquez DM, et al. Smoking and smoking cessation among persons with severe mental illness. Psych Serv 2006;57:462.

11. Baxter DN. The mortality experience of individuals on the Salford psychiatric case register I. Br J Psych 1996;168:772-9.

12. Brown S, Birtwistle LR, Thompson C. The unhealthy lifestyle of people with schizophrenia. Psychol Med 1999;29:697-701.

13. Els C. Canada: pharmacy tobacco sales to mentally ill people. Tob Control 2007;16:75.

14. Olivier D, Lubman DI, Fraser R. Tobacco smoking within psychiatric inpatient settings: biopsychosocial perspective. Austr NZ J Psych 2007:41:572-80.
15. Glassman AH. Cigarette smoking: implication for psychiatric illness. Am J Psych 1993:150:546-53.

16. Leonard S, Adler LE, Benhammou K, et al. Smoking and mental illness. Pharmacol Biochem Behav 2001:70:561-70.

17. Kumari V, Postma P. Nicotine use in schizophrenia: the self medication hypotheses. Neurosci Biobehav Rev 2005;29:1021-34.

18. Lyon ER. A review of the effects of nicotine on schizophrenia and antipsychotic medications. Psych Serv 1999;50:1346-50.

19. Procyshyn RM, Tse G, Sin 0, et al. Concomitant clozapine reduces smoking in patients treated with risperidone. Eur Neuropsychopharmacol 2002;12:77-80.

20. George TP, Sernyak MJ, Ziedonis DM, et al. Effects of clozapine on smoking in chronic schizophrenic patients. J Clin Psych 1995:56:344-6.

21. Leas $\mathbf{L}, \mathbf{M c C}$ abe $\mathbf{M}$. Health behaviours among individuals with schizophrenia and depression. J Health Psychol 2007;12:563-79.

22. El-Guebaly N, Cathcart J, Currie S, et al. Smoking cessation approaches for persons with mental illness or addictive disorders. Psych Serv 2002;53:1166-71.

23. Chou KR, Chen R, Lee JF, et al. The effectiveness of nicotine-patch therapy for smoking cessation in patients with schizophrenia. Int J Nursing Stud 2004;41: 321-30.

24. Addington J. Group treatment for smoking cessation among persons with schizophrenia. Psych Serv 1998;49:925-8.

25. Goldberg J0, Moll S, Washington A. Exploring the challenge of tobacco use in schizophrenia. Psych Rehab Skills 1996;1:51-63.

26. Whelton C, Pawlick J, Cook PE. Growing with people with a psychiatric disability in a psychosocial rehabilitation program. Psych Rehab 1999:22:290-3.

27. Dermer SW, Landeen JL. Establishing a model for care in schizophrenia: one program's experience. Can J Psych 1991;36:588-93.

28. Mueser KT, Bond GR, Drake RE, et al. Models of community care for severe mental illness: a review of research on case management. Schizophrenia Bull 1998;24: $37-74$.

29. Mills C, Stephens T, Wilkins K. Summary report of the workshop on data for monitoring tobacco use. Chron Dis Can 1994;15:105-110.

\section{Submit an eletter, and join the debate}

eLetters are a fast and convenient way to register your opinion on topical and contentious medical issues. You can find the "submit a response" link alongside the abstract, full text and PDF versions of all our articles. We aim to publish swiftly, and your comments will be emailed directly to the author of the original article to allow them to respond. eLetters are a great way of participating in important clinical debates, so make sure your voice is heard. 